\title{
Behandlungsempfehlung
}

\section{Individuelle Therapie der Colitis ulcerosa}

\author{
Beim akuten Schub der Colitis ulcerosa heißt es: je nach Befallsmuster therapieren! \\ Dafür steht ein breites Spektrum an Behandlungsoptionen zur Verfügung.
}

\begin{abstract}
Den Weg zur optimalen Therapie bei Colitis ulcerosa weisen nach wie vor Befallsmuster und Krankheitsaktivität. „Eine adäquate Lebensqualität und eine steroidfreie Remission sind realistische Ziele, gegebenenfalls auch eine Abheilung der mukosalen Entzündung", schreiben Dr. Sarah Fischer vom Uniklinikum Erlangen und Kollegen [1]. Die Therapieempfehlungen in ihrem Update zur Colitis ulcerosa basieren auf den aktuellen deutschen und europäischen Leitlinien [2 und 3].
\end{abstract}

\section{Leicht- bis mäßiggradige Proktitis}

Bei leicht- bis mäßiggradiger Proktitis kommen laut Autoren zunächst 5-Aminosalicylate (5-ASA) als Suppositorien zum Einsatz. Spricht der Patient nicht auf die Therapie an, sollte eine Kombi-Therapie mit oralen 5-ASA-Präparaten (etwa Salofalk ${ }^{\circ}$, Claversal ${ }^{\circ}$, Mezavant ${ }^{\circ}$, Pentasa ${ }^{\circ}$ )

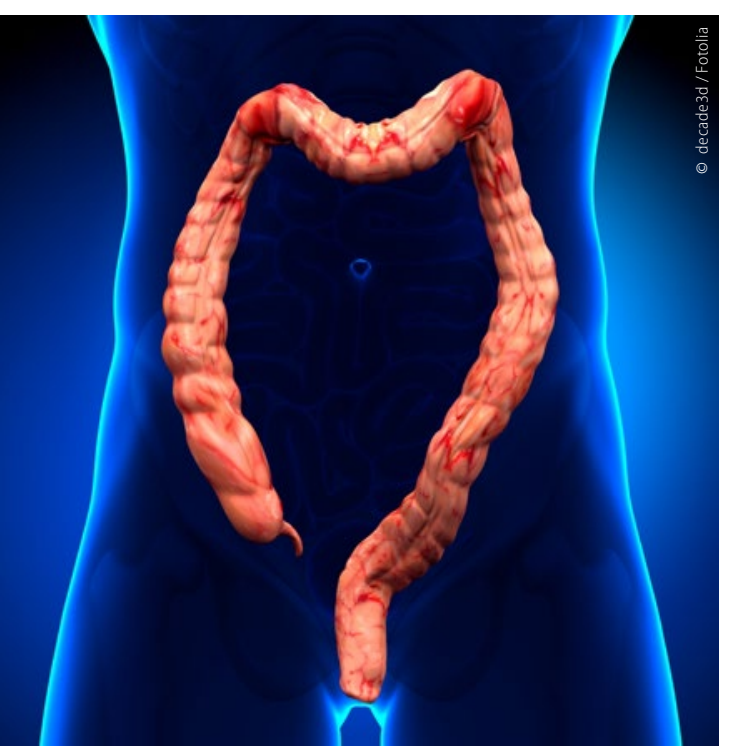

Bei leicht- bis mäßiggradiger Pankolitis werden zunächst orale und rektale 5-ASA-Präparate kombiniert. oder topischen Steroiden wie Budesonid durchgeführt werden. Ist die Proktitis therapierefraktär, können auch Immunsuppressiva und/oder Biologika notwendig werden.

\section{Leicht- bis mäßiggradige \\ Linksseitenkolitis}

Wenn die Entzündung das Rektum überschreitet, sollten 5-ASA bei leichter oder mäßig schwerer Entzündung schon zur initialen Therapie oral und rektal (Klysmen, Rektalschäume) appliziert werden. Bei Patienten, die auf diese Therapie klinisch nicht ansprechen, kann laut Autoren für einen Zeitraum von acht Wochen zusätzlich zum 5-ASA-Präparat Budesonid in einer Multimatrix (MMX)-Formulierung (Cortiment ${ }^{\oplus} \mathrm{MMX}^{\circledast}$ ) eingesetzt werden. Alternativ und insbesondere dann, wenn die Entzündung ausgeprägt und der Verlauf refraktär ist, ist eine systemische Steroidtherapie indiziert. Die Kortikosteroide kommen aber nur zur Remissionsinduktion in Betracht.

\section{Pankolitis}

Ist das gesamte Kolon leicht bis mäßiggradig befallen, wird mit einer Kombinationstherapie aus 5-ASA-Präparaten zur oralen und rektalen Anwendung in Form von Klysmen oder Rektalschäumen therapiert. Ist die Entzündung nicht rückläufig, sind orale Kortikosteroide indiziert; diese sollten aber - abhängig vom klinischen Ansprechen - innerhalb von acht bis zwölf Wochen ausgeschlichen werden, schreiben Fischer und Kollegen.

Bei steroidabhängigem Verlauf ist eine Thiopurin-Therapie notwendig. Therapiealternativen sind die TNF-Antikörper Adalimumab (Humira ${ }^{\circledR}$ ), Infliximab (Remicade ${ }^{\circledast}$, von dem es mittlerweile auch Biosimilars gibt, und Golimumab (Simponi ${ }^{\circledast}$ ) oder, bei Patienten, bei denen kon- ventionelle Arzneien und andere Biologika versagt haben, der AdhäsionsmolekülAntikörper Vedolizumab (Entyvio ${ }^{\varpi}$ ).

\section{Schwerer Colitis-ulcerosa-Schub}

Ein schwerer Schub ist gekennzeichnet durch $\geq 6$ blutige Diarrhöen pro Tag, Fieber, Tachykardie, Anämie und einer Blutsenkungsgeschwindigkeit $\geq 30 \mathrm{~mm} / \mathrm{h}$. In solchen Fällen sollte eine intravenöse Steroidtherapie unter stationären und interdisziplinären Bedingungen erfolgen. Ist die Colitis in drei bis fünf Tagen unter dieser Therapie nicht rückläufig, werden Ciclosporin A oder TNF-Antikörper eingesetzt. „Bei den Therapieentscheidungen sollte man zu jedem Zeitpunkt auch kritisch die mögliche Notwendigkeit zur Proktokolektomie evaluieren“, schreiben Fischer und Kollegen.

Entscheidet sich ein Patient gegen eine OP, ist ein Wechsel des Therapieprinzips von TNF-Antikörpern zu CalcineurinInhibitoren beziehungsweise vice versa möglich, schreibt Prof. Dr. Andreas Stallmach vom Uniklinikum Jena im Handbuch Gastroenterologie 2016. Die Wahrscheinlichkeit des Ansprechens und des Darmerhalts betrage wohl mehr als 50\%, es seien allerdings schwere Nebenwirkungen zu beachten. Die Drittlinien-Therapie sei mit einer signifikanten Mortalitätsrate verknüpft und sollte daher Behandlungszentren vorbehalten bleiben, schreibt Stallmach.

Fischer und Kollegen erinnern in ihrem Beitrag, dass bei schweren oder therapierefraktären Verläufen immer auch an eine pseudomembranöse Colitis oder eine Zytomegalievirus-Infektion gedacht werden muss.
MMW 2016; 158: 77-82

2. Z Gastroenterol 2011; 49: 1276-1341

3. J Crohns Colitis 2012; 6: 991-1030 